\title{
Valor de las pruebas inmunológicas en el diagnóstico de las enfermedades micóticas: experiencia de un centro de referencia
}

\author{
Magaly Chinchilla ${ }^{1}$, Marta Cecilia Kogson ${ }^{2}$, Ruth de Insignares ${ }^{3}$, María Claudia Rodríguez ${ }^{4}$, \\ Martha Vargas ${ }^{5}$, Edilma Torrado ${ }^{1}$, Elizabeth Castañeda ${ }^{1}$
}

\begin{abstract}
Resumen
Las pruebas inmunológicas han sido ampliamente utilizadas como evidencia indirecta de infección micótica y tienen valor diagnóstico; algunas de estas pruebas pueden ser fácilmente adaptadas en los laboratorios con un bajo nivel de complejidad. El Laboratorio de Micología del Grupo de Microbiología del Instituto Nacional de Salud es centro de referencia para el diagnóstico de las micosis sistémicas en el país; por tal motivo y con el fin de establecer una red nacional, se seleccionaron cuatro laboratorios regionales y se entrenó personal en técnicas inmunológicas para realizar el diagnóstico de la histoplasmosis y de la paracoccidioidomicosis. Las técnicas empleadas fueron: inmunodifusión (ID) para histoplasmosis, paracoccidioidomicosis y aspergilosis; fijación de complemento (FC) para histoplasmosis y paracoccidioidomicosis y, aglutinación con partículas de látex para la antigenemia en la criptococosis. En un período de 7 años (1990 -1996), los laboratorios procesaron 6.841 muestras de suero correspondientes a 6.411 pacientes con diagnósticos compatibles con micosis sistémicas. Los cuatro laboratorios regionales analizaron 1.918 muestras (28\%) y el centro de referencia, 4.923 (72\%). La reactividad total fue de 13,5\% (923/6.841), discriminada así: histoplasmosis, $42,5 \%$; paracoccidioidomicosis, $33 \%$; criptococosis, $21,2 \%$, y aspergilosis, 3,3\%. El número total de casos diagnosticados fue de $441: 155$ histoplasmosis, 120 paracoccidioidomicosis, 138 criptococosis y 28 aspergilosis. El empleo de la ID en los centros regionales permitió el diagnóstico de $31 \%$ de los pacientes con histoplasmosis, de $41,6 \%$ de los pacientes con paracoccidioidomicosis y $53,6 \%$ de los pacientes con aspergilosis, lo cual indica que el diagnóstico precoz se puede realizar a nivel regional. La sensibilidad y la simplicidad de la prueba de ID constituye un excelente recurso para los laboratorios de baja complejidad, a nivel regional, que operan en áreas endémicas.
\end{abstract}

\footnotetext{
1 Grupo de Microbiología, Instituto Nacional de Salud, Santa Fe de Bogotá, D.C.

2 Hospital Santa Sofía, Manizales, Caldas.

3 Centro de Rehabilitación Fernando Troconis, Santa Marta, Magdalena.

4 Hospital Erasmo Meoz, Cúcuta, Norte de Santander.

5 Hospital San Antonio, Villavicencio, Meta.

Recibido para su publicación: 1 de junio de 1998 - Aprobado para su publicación: 30 de junio de 1998
} 


\section{The value of immunological tests in mycotic disease diagnosis:a reference centre's experiences.}

Immunological tests have been widely used as indirect evidence of mycotic infection and have diagnostic value. Some of these tests can be easily adapted to be used in laboratories having a lower level of complexity. The National Institute of Health's Microbiology Group's Micology Laboratory is a reference centre for the diagnosis of systemic mycoses in Colombia. Thus, and having the intention of establishing a national network, four regional laboratories were selected and their personnel trained in immunological techniques so that they could carry out histoplasmosis and paracoccidioidomycosis diagnoses. The techniques employed were immunodifusion (ID), for histoplasmosis, paracoccidioidomycosis and aspergillosis, complement fixation (CF) for histoplasmosi and paracoccidioidomycosis and latex agglutination tests for antigenaemia in cryptococosis.

In a period of 7 years (1990-19976), the laboratories processed 6,841 sera samples, corresponding to 6,411 patients having diagnoses compatible with systemic mycosis. The four regional laboratories analysed $1,918(28 \%)$ samples and the reference centre $4,923(72 \%)$. Total reactivity was $13.5 \%(923 / 6,841)$, broken down as follows: histoplasmosis $42.5 \%$, paracoccidioidomycosis $33 \%$, cryptococosis $21.2 \%$ and aspergillosis $3.3 \%$. The total number of diagnosed cases was 441: 155 histoplasmosis, 120 paracoccidioidomycosis, 138 cryptococcosis and 28 aspergillosis. The use of the ID technique in the regional centres allowed the diagnosis of $31 \%$ of patients as having histoplasmosis, $41.6 \%$ having paracoccidoidomycosis and $53.6 \%$ as having aspergillosis, which indicates that early diagnosis can be carried at regional level. The ID test sensitivity and simplicity constitute an excellent resource for those laboratories having less complexity, at regional level, which operate in endemic areas.

El diagnóstico de las enfermedades micóticas se realiza con base en pruebas de laboratorio, ya sea en forma directa como lo son la visualización y recuperación del agente etiológico a partir de muestras clínicas o en forma indirecta, como son la detección de antígenos o de anticuerpos en líquido cefalorraquídeo o en sueros (1-3). Estas últimas pruebas han sido ampliamente empleadas como evidencia de infección micótica y, dada su sensibilidad y especificidad, tienen valor diagnóstico (1-3).

Entre nosotros, para el diagnóstico de la paracoccidioidomicosis y de la histoplasmosis, las pruebas empleadas para detectar anticuerpos son la inmunodifusión (ID) con una sensibilidad de $97 \%$ y $72 \%$, respectivamente, y la fijación de complemento (FC) con una sensibilidad de $95 \%$ y $83 \%$ para estas dos entidades $(2,3)$. En el diagnóstico de la criptococosis, se utiliza la aglutinación con partículas de látex para detectar el antígeno polisacárido de Cryptococcus neoformans, lo cual se logra con una sensibilidad de $94 \%$ en muestras de suero y una especificidad, en nuestra experiencia, de $100 \%$ (4).

La importancia de las pruebas inmunológicas en las infecciones micóticas se ha comprobado por la ayuda que prestan en el diagnóstico de casos primarios o de difícil resolución, en los cuales las pruebas directas han sido negativas. Además, los niveles de antígeno o de anticuerpos permiten el seguimiento de los pacientes durante su tratamiento $(1,4)$.

El Laboratorio de Micología del Grupo de Microbiología del INS es un centro de referencia para el diagnóstico de las micosis sistémicas; por esta razón, se ha establecido un grupo de trabajo compuesto por laboratorios nacionales, con los cuales se han implementado algunas pruebas serológicas estandarizadas de fácil adaptación a laboratorios de baja complejidad. Con el fin de establecer un programa de 
inmunodiagnóstico de micosis sistémicas, se seleccionaron 4 laboratorios que atendían pacientes sintomáticos respiratorios y su personal se entrenó en las técnicas serológicas para el diagnóstico de paracoccidioidomicosis, histoplasmosis y aspergilosis. Las actividades de estos laboratorios y del centro de referencia durante 7 años (1990-1996) son el objeto del presente informe.

\section{Materiales y métodos}

\section{Muestras}

Entre 1990 y 1996, se analizaron 6.841 sueros provenientes de 6.411 pacientes que fueron remitidos al Laboratorio de Micología del Grupo. De ellos, 1.918 fueron procesados inicialmente en los 4 laboratorios regionales, 383 en el Hospital Santa Sofía de Manizales, 432 en el Centro de Rehabilitación Fernando Troconis de Santa Marta, 747 en el Hospital San Antonio de Villavicencio y 356 en el Hospital Erasmo Meoz de Cúcuta.

\section{Pruebas serológicas}

1. Inmunodifusión (ID). Se empleó la técnica de ID para determinar anticuerpos anti- $H$. capsulatum, anti- $P$. brasiliensis y antiAspergillus fumigatus, $A$. flavus y $A$. niger. La prueba se realizó en agarosa al $1 \%$ con $0,85 \%$ de $\mathrm{NaCl}$ y $0,1 \%$ de azida de sodio (5). Los antígenos empleados fueron la paracoccidioidina, antígeno metabólico de levadura de $P$. brasiliensis preparado por el método descrito por Camargo (6); la histoplasmina, antígeno metabólico de la fase miceliar de $H$. capsulatum var. capsulatum preparado según el método de Harrel (7) y las aspergilinas, antígenos metabólicos de $A$. fumigatus, $A$. flavus y $A$. niger preparados por el método descrito por Palmer (5). Los antígenos producidos en el laboratorio y los sueros control fueron distribuidos a los centros regionales.

2. Fijación de complemento (FC). Se empleó la técnica de FC para determinar anticuerpos anti- $H$. capsulatum y anti- $P$. brasiliensis. Se realizó la técnica del $50 \%$ de hemólisis (8) con los antígenos metabólicos de fase de levadura de $P$. brasiliensis (6) y de fase miceliar de $H$. capsulatum var. capsulatum (7). Así mismo, el antígeno compuesto por blastoconidias completas de este último hongo (7).

3. Aglutinación. Se determinó el antígeno polisacárido de $C$. neoformans con el empleo de la prueba de aglutinación de partículas de látex recubiertas con anticuerpos anti-C. neoformans (Cryptococcal antigen latex agglutination system, Meridian Diagnostics, Cincinnati, Ohio, E.U.) (5).

En los laboratorios regionales se realizó la ID y en el centro de referencia, la ID, la FC y la aglutinación de látex.

\section{Resultados}

\section{Grupo total}

Se observó una reactividad global de 13,5\% (923/6.841) de los sueros analizados. Los porcentajes de reactividad, según la procedencia, fueron de 20\% (76/383) para Caldas, 17\% (73/432) para Magdalena, 14\% (104/747) para Meta y $18 \%$ (64/356) para Norte de Santander. En el centro de referencia se observó una reactividad de 12,3\% (606/4.923).

\section{Reactividad según la entidad}

En el cuadro 1 se consigna la reactividad por entidad y por laboratorio. Los anticuerpos contra H. capsulatum var. capsulatum se determinaron con una frecuencia mayor de $37 \%$ en Caldas, Meta y Norte de Santander. Por el contrario, la paracoccidioidomicosis se diagnosticó con mayor frecuencia en Magdalena (97\%) y Norte de Santander (63\%). En el cuadro 2 se presenta la reactividad total en las pruebas inmunológicas por laboratorio y por micosis. Los porcentajes de reactividad total fueron de $42,5 \%$ para la histoplasmosis, 33\% para la paracoccidioidomicosis, $21,2 \%$ para la criptococosis y $3,3 \%$ para la aspergilosis.

El número total de casos diagnosticados fue de 441: 155 histoplasmosis, 120 paracoccidioidomicosis, 138 criptococosis y 28 aspergilosis. Los laboratorios regionales diagnosticaron 48 (31\%) casos de histoplasmosis, $50(41,6 \%)$ casos de paracoccidioidomicosis y $15(53,6 \%)$ casos de aspergilosis. 
Cuadro 1. Reactividad de las pruebas inmunológicas por laboratorio y por entidad.

\begin{tabular}{|c|c|c|c|c|c|c|c|c|c|c|}
\hline \multirow[t]{2}{*}{ Entidad } & \multicolumn{2}{|c|}{$\begin{array}{l}\text { Caldas } \\
(n=383)\end{array}$} & \multicolumn{2}{|c|}{$\begin{array}{l}\text { Magdalena } \\
(n=432)\end{array}$} & \multicolumn{2}{|c|}{$\begin{array}{c}\text { Meta } \\
(\mathrm{n}=747)\end{array}$} & \multicolumn{2}{|c|}{$\begin{array}{l}\text { N.Santander } \\
(n=356)\end{array}$} & \multicolumn{2}{|c|}{$\begin{array}{l}\text { Centro de referencia } \\
\qquad(n=4.923)\end{array}$} \\
\hline & $n$ & $\%$ & $\mathrm{n}$ & $\%$ & $n$ & $\%$ & $n$ & $\%$ & $n$ & $\%$ \\
\hline Histoplasmosis & 37 & 49 & 2 & 3 & 48 & 46 & 24 & 37 & 281 & 46 \\
\hline Paracoccidioidomicosis & 24 & 31 & 71 & 97 & 56 & 54 & 40 & 63 & 113 & 19 \\
\hline Aspergilosis & 15 & 20 & 0 & 0 & 0 & 0 & 0 & 0 & 15 & 2,5 \\
\hline Criptococosis & 0 & 0 & 0 & 0 & 0 & 0 & 0 & 0 & 197 & 32,5 \\
\hline Total & 76 & 100 & 73 & 100 & 104 & 100 & 64 & 100 & 606 & 100 \\
\hline
\end{tabular}

Cuadro 2. Reactividad total en las pruebas inmunológicas por laboratorio y por micosis.

\begin{tabular}{lcccrr}
\hline Laboratorio & Histoplasmosis & Paracoccidioidomicosis & Criptococosis & Aspergilosis & Total \\
\hline $\begin{array}{l}\text { Regional }(n=4) \\
\begin{array}{l}\text { Centro de } \\
\text { referencia }(n=1)\end{array}\end{array}$ & 111 & 191 & No realizado & 15 & 317 \\
\hline Total & $392(42,5 \%)$ & 113 & 197 & 15 & 606 \\
\hline
\end{tabular}

*53 y **147 muestras previamente publicadas $(1,4)$

\section{Discusión}

Usualmente, las micosis sistémicas no son consideradas en pacientes sintomáticos con enfermedad pulmonar, debido a su semejanza con la tuberculosis, enfermedad altamente endémica en nuestro país (9). El hábitat de los agentes etiológicos de la histoplasmosis y de la criptococosis ha sido determinado en diversos países incluyendo a Colombia $(10,11)$; el del agente de la paracoccidioidomicosis aún no ha sido determinado con exactitud, pero el consenso es que el hongo se encuentra en los suelos del área endémica (12) y se conoce la ubicuidad de las especies de Aspergillus, agentes de las aspergilosis (13). Por lo tanto, la población general puede estar expuesta a la infección natural con estos agentes micóticos. De hecho, estas infecciones son de amplia distribución a nivel mundial y pueden dar origen a enfermedad clínicamente manifiesta. Es por ello que las pruebas de laboratorio constituyen una valiosa ayuda para establecer el diagnóstico diferencial en estos pacientes $(1,4)$.

La sensibilidad y especificidad de las pruebas aplicadas al diagnóstico micológico han sido ampliamente documentadas (1-4) y, dada su efectividad, se emplean como herramientas diagnósticas ya que suministran una evidencia indirecta de infección.

Previo al análisis de los datos, es importante recordar que los pacientes que ingresaron al estudio a través de los laboratorios regionales constituyen una población seleccionada, sintomáticos respiratorios en quienes se había considerado la tuberculosis pulmonar como diagnóstico diferencial. Por tanto, el hallazgo de una reactividad de 14 a $20 \%$ de los sueros estudiados refleja la selección de la población.

La reactividad observada en las pruebas inmunológicas realizadas en los cuatro laboratorios regionales y en el centro de referencia, demuestran la frecuencia de la paracoccidioidomicosis y de la criptococosis como enfermedades clínicamente manifiestas en nuestro medio $(1,4,14,15)$. Es importante resaltar el número de pacientes con paracoccidioidomicosis diagnosticados en el departamento del Magdalena; la revisión de la historia de estos pacientes señala que son procedentes de la región de la Sierra Nevada de Santa Marta o que han realizado actividades laborales en la misma, lo cual la demarcaría como una reserva 
para la entidad, semejante a las que se han descrito en las regiones cafeteras, endémicas de Colombia (1) y de Latino américa (2).

Ha sido posible diagnósticar histoplasmosis, como infección asintomática o en las formas clínicas agudas y diseminadas. La ID y la FC han sido invaluables en el estudio de diversos brotes de histo-plasmosis $(10,16)$ y los laboratorios regionales han jugado un papel muy importante en el diagnóstico del caso índice que exhibe la forma clínica de la histoplasmosis aguda, así como también el de los pacientes infectados pero asintomáticos. Por su parte, la FC realizada en el laboratorio central también permitió un seguimiento adecuado de los pacientes reactivos.

De los datos presentados, se destaca la mayor frecuencia de sueros reactivos en la histoplasmosis, lo cual señala la frecuencia de infecciones por $H$. capsulatum var. capsulatum en el país. Ello permite afirmar que la histoplasmosis es la micosis endémica más importante en nuestro medio, afirmación que cobra importancia al considerar que en el área endémica de los Estados Unidos, la histoplasmosis diseminada en pacientes infectados con el VIH es de gran importancia (17).

La importancia de la paracoccidioidomicosis en nuestro medio ha sido señalada anteriormente con base en el número de casos diagnosticados e informados en la literatura $(1,2,14,18)$. Los 120 casos descritos en esta serie así lo confirman; vale la pena mencionar que 53 de estos casos han sido publicados $(1,18)$.

La aglutinación con partículas de látex es una excelente prueba empleada en el diagnóstico de la criptococosis, puesto que detecta antígeno circulante, esta prueba es una valiosa ayuda en el diagnóstico y en el seguimiento de los pacientes bajo tratamiento (4).

La sensibilidad y simplicidad de la ID con los diferentes antígenos empleados, la convierten en un excelente recurso para el diagnóstico de las enfermedades micóticas a nivel regional, en áreas endémicas.

\section{Referencias}

1. Castillo J, Ordóñez N, López S, Castañeda E. Paracoccidioidomicosis. Diagnóstico por el laboratorio de 333 casos. Biomédica 1994;14:230-9.

2. Brummer E, Castañeda E, Restrepo A. Paracoccidioidomycosis: an update. Clin Microbiol Rev 1993;6:89-117.

3. Wheat LJ. Diagnosis and management of histoplasmosis. Eur J Clin Microbiol Infect Dis 1989; 8:480-90.

4. Ordóñez N, Castañeda E, Torrado E. Criptococosis meníngea de 1990 a 1995 . Hallazgos del laboratorio. Biomédica 1996;16:93-7.

5. PaImer DF, Kaufman L, Kaplan W, Cavallaro JJ. In: Serodiagnosis of mycotic disease. Springfield, III. Charles C Thomas. 1977.

6. Camargo ZP, Unterkircher C, Campoy SP, Travassos LR. Production of Paracoccidioides brasiliensis exoantigens for immunodiffussion tests. J Clin Microbiol 1988;26:2147-51.

7. Harrel, W, Ashworth H, Britt LE, George JR, Gray SB, Green JH et al. Specifications and evaluation methods for immunological and microbiological reagents bacterial, fungal, parasitic manual. Department of Health, Education and Welfare. Public Health Service. Center for Disease Control. Atlanta. Volume 1. 1973: 107-11.

8. Kaufmann L, Huppert M, Fava-Netto C, Negroni R, Restrepo A. Manual of standardized serodiagnostic procedures for systemic mycosis. Part II: complement fixation test. Washington, D.C.: Panamerican Health Organization, 1972.

9. Garzón MC, Mejía S, Sierra CR. Evaluación del programa de control de la tuberculosis del Laboratorio Nacional de Referencia, Colombia, 19921995. IQEN 1997; 2:122-24.

10. Ordóñez N, Tobón A, Arango M, Tabares A, De Bedout C, Gómez B, Castañeda E, Restrepo A. Brotes de histoplasmosis registrados en el área andina colombiana. Biomédica 1997;17:105-11.

11. Ordóñez N, Rodríguez MC, Callejas A, Castañeda E. Search for the habitat of Cryptococcus neoformans var. gattii in the northeast city in Colombia. Preliminary data. $13^{\text {th }}$ Congress of the International Society for Human and Animal Mycology. Parma, Italy. 1997; P-109:105.

12. Wanke B, Londero AT. Epidemiology and infection. In: Paracoccidioidomycosis. Marcelo Franco, Carlos da Silva Lacas, Angela Restrepo, Editors paracoccidioidomycosis CRC, 1994:109-20.

13. Lentino JR, Rosenkranz MA, Micheals JA, Kurup VP, Rose HD, et al. Nosocomial aspergillosis: a retrospective review of airborne disease 
secondary to road construction and contaminated air conditioners. Am J Epidemiol 1982;116:335-341.

14. Spir N. Paracoccidioidomicosis: revisión retrospectiva de 100 pacientes. Rev CES Med 1991; 5:125130.

15. Lizarazo J, Rodríguez MC, Ordóñez N, Vargas JJ, Castañeda E. Criptococosis meníngea en el Hospital Erasmo Meoz de Cúcuta. Acta Neurol Colomb 1995; 11:259-67.

16. Rodríguez MC, Arango M, Díaz S, Tobón A, Restrepo A, Chinchilla M, Castañeda E. Brotes y casos de histoplasmosis, Colombia, 1995. IQCB 1996;2:50-4.

17. Wheat LJ, Connolly-Stringfield P, Baker RL, Curfman MF, Eads ME, Israel KS, et al. Disseminated histoplasmosis in the acquired immune deficiency syndrome: clinical finding, diagnosis and treatment, and review of the literature. Medicine 1990;69:361-74.

18. Castañeda E, Ordóñez N, Bustos L, Sánchez J. Paracoccidioidomicosis. Diagnóstico por el laboratorio de 87 casos. Acta Med Colomb 1981;6:339-48. 